\title{
The Japanese Society of Hypertension Guidelines for the Management of Hypertension (JSH 2009) - Chapter 2
}

Toshio Ogihara, Kenjiro Kikuchi, Hiroaki Matsuoka, Toshiro Fujita, Jitsuo Higaki, Masatsugu Horiuchi, Yutaka Imai, Tsutomu Imaizumi, Sadayoshi Ito, Hiroshi Iwao, Kazuomi Kario, Yuhei Kawano, Shokei Kim-Mitsuyama, Genjiro Kimura, Hiroaki Matsubara, Hideo Matsuura, Mitsuhide Naruse, Ikuo Saito, Kazuyuki Shimada, Kazuaki Shimamoto, Hiromichi Suzuki, Shuichi Takishita, Norio Tanahashi, Takuya Tsuchihashi, Makoto Uchiyama, Shinichiro Ueda, Hirotsugu Ueshima, Satoshi Umemura, Toshihiko Ishimitsu and Hiromi Rakugi, on behalf of The Japanese Society of Hypertension Committee

Hypertension Research (2009) 32, 318; doi:10.1038/hr.2009.34

Correction to: Hypertension Research (2009) 32, 11-23; doi:10.1038/ hr.2008.2

A machine number specified in the JSH Guidelines (Chapter 2, page 15) was incorrect.

On page 15, in the paragraph referring to the measurement of central blood pressure and augmentation index (AI) from radial artery pulse waves, the device 'Omron Healthcare HEM7000AI' was stated as being used. This should have read HEM9000AI.
The corrected sentence should therefore read:

In Japan, a device for measuring central blood pressure and AI from radial artery pulse waves (Omron Healthcare, HEM9000AI) is used. 